\title{
Comparison of open and laparoscopic inguinal hernia repair in octogenarians at a single center
}

\author{
Xiaoqiang Zhu ${ }^{1}$, Zhengni Liu ${ }^{1}$, Jianfeng Shen ${ }^{1}$, Jiajie Liu ${ }^{1}$, and Rui Tang ${ }^{1}$ \\ ${ }^{1}$ Shanghai East Hospital
}

November 9, 2020

\begin{abstract}
Aim: Although the advantages of laparoscopic inguinal hernia repair in the general population have been shown, its role in octogenarians has yet to be elucidated. This retrospective study compared laparoscopic and open inguinal hernia repair in octogenarians; the experience was summarized to aid in selecting surgical and anesthesia options in this age group. Materials and methods: The data of octogenarians who underwent laparoscopic $(\mathrm{n}=81)$ or open $(\mathrm{n}=121)$ inguinal hernia repair in our center from January 2017 to December 2019 were collected. Statistical analysis variables included basic epidemiological data of patients, anesthesia methods, surgical procedures, comorbidities, postoperative pain, complications, recurrence, and other data. Results: There were no significant differences between the two groups in terms of sex, body mass index, recurrent hernias, comorbidities, postoperative complications, and recurrence. The American Society of Anesthesiologists (ASA) class and the proportion of scrotal hernias in the open group were higher than those of the laparoscopic group, whereas the proportion of bilateral hernias in the laparoscopic group was higher than that in the open group. The postoperative pain scores of the laparoscopic group were lower than those of the open group. The proportion of patients with ASA class III-IV was highest in patients receiving local anesthesia, and the proportion of bilateral hernias was higher in the patients receiving general anesthesia than that in those receiving local or spinal anesthesia. Conclusion: In octogenarians, both laparoscopic and open inguinal hernia repair are safe and feasible, but an appropriate surgery and anesthesia plan should be determined according to the patient's specific conditions.
\end{abstract}

Title: Comparison of open and laparoscopic inguinal hernia repair in octogenarians at a single center

\section{Short running title: Inguinal hernia repair in octogenarians}

Xiaoqiang Zhu ${ }^{\mathrm{a}}$, Zhengni Liu ${ }^{\mathrm{a}}$, Jianfeng Shen ${ }^{\mathrm{a}}$, Jiajie Liu ${ }^{\mathrm{a}}$, Rui Tanga, *

a Department of Hernia and Abdominal Wall Surgery, Shanghai East Hospital, Tongji University, 150 Jimo Road, Shanghai 200120, China

* Corresponding author : Rui Tang, PhD, MD

Department of Hernia and Abdominal Wall Surgery

Shanghai East Hospital, Tongji University

150 Jimo Road, Shanghai 200120, China

Tel: +86 13817151221

Fax: +86 2138804518

E-mail: kevintown@126.com 
Funding: This study was supported by Shanghai East Hospital [No. DFRC2017009].

\section{Acknowledgments}

The authors would like to thank Enago (www.enago.cn) for the English language review

Declaration of interest: None.

\section{Hosted file}

main text file.pdf available at https://authorea.com/users/374125/articles/491737-comparison-

of-open-and-laparoscopic-inguinal-hernia-repair-in-octogenarians-at-a-single-center 